\title{
Modelamiento del crecimiento urbano de la ciudad de Huaraz mediante regresión logística
}

\author{
Huaraz city urban growth modeling through logistic regression
}

\author{
Ricardo Ray Villanueva Ramírez ${ }^{1}$, Carlos Del Valle Jurado ${ }^{2}$
}

Recibido:10/07/2020 - Aprobado: 18/11/2020 - Publicado: 18/06/2021

\begin{abstract}
RESUMEN
En 1941, la ciudad de Huaraz fue afectada por un aluvión originado en la laguna Palcacocha, de origen glaciar, desde entonces la ciudad creció significativamente ocupando áreas expuestas a aluviones. Para analizar futuros riesgos es necesario evaluar prospectivamente el crecimiento de la cuidad siendo la regresión logística una técnica ampliamente empleada. Mediante herramientas geomáticas, se corrigieron visual y geométricamente fotografías aéreas y satelitales de 1948, 1962, 1977, 2011 y 2018 tomando como cartografía base el Plano Catastral 1/10,000. Con trabajo de campo complementario se obtuvieron los polígonos urbanos/poblados en una zona de análisis de $25.6 \mathrm{~km}^{2}$, con los que se analizó la tendencia espacial histórica y características de crecimiento en relación con factores como pendiente, altitud, distancia al centro medio de la ciudad, dirección de crecimiento y densidad de puntos; que fueron empleados como variables independientes dentro del modelo de regresión logística siendo la dependiente la cobertura urbana/poblada del 2018. Entre 1948 y 2018 Huaraz creció 598\% en dirección Sur-Norte con sesgo hacia el Este. Excluyendo el área urbana/poblada del 2018, el $21 \%$ de la zona de análisis, posee probabilidades superiores a 0.2 de albergar nuevas áreas urbanas/pobladas, predominando las probabilidades de 0.2 a 0.4 con el $11 \%$.
\end{abstract}

Palabras clave: Crecimiento urbano; regresión logística; geomática; tendencia espacial; Huaraz.

\begin{abstract}
In 1941, Huaraz city was affected by a flood from Palcacocha glacier lagoon, thenceforth the city grew significantly occupying areas exposed to floods. For future risks analysis, city growth prospective evaluation is necessary, being logistic regression a widely used technique. Using geomatic tools, aerial and satellite photographs from 1948, 1962, 1977, 2011 and 2018 were visually and geometrically corrected taking cadastral map 1:10,000 as base cartography. With complementary field work polygons of urban/populated areas were obtained within a $25 \mathrm{~km}^{2}$ analysis zone, with which the historical spatial trend and growth characteristics were analyzed in relation to variables such slope, altitude, distance from city middle center, direction growth and point density; which were used as independent variables in the regression logistic model being the dependent the 2018 urban/populated coverage. Between 1948 and 2018 Huaraz grew 598\% in South-North direction with bias towards East. Excluding 2018 urban/populated area, 21\% of the analysis zone has probabilities of housing new urban/populated areas greater than 0.2 , prevailing 0.2 to 0.4 probabilities with $11 \%$.
\end{abstract}

Keywords: Urban growth; logistic regression; geomatic; spatial trend; Huaraz.

1 Universidad Nacional Mayor de San Marcos, Facultad de Ingeniería Geológica, Minera, Metalúrgica y Geográfica, Unidad de Posgrado. Avenida Venezuela s/n Cuadra 34 Ciudad Universitaria, Cercado de Lima. E-mail: rvillanuevar@unasam.edu.pe - ORCID: https:/lorcid.org/0000-0001-6049-2906

2 Universidad Nacional Mayor de San Marcos, Facultad de Ingeniería Geológica, Minera, Metalúrgica y Geográfica, EP Ing. Geológica. Avenida Venezuela s/n Cuadra 34 Ciudad Universitaria, Cercado de Lima. E-mail: cdelvallej@unmsm.edu.pe - ORCID: https://orcid.org/0000-0002-3040-1396 


\section{INTRODUCCIÓN}

Nos encontramos a nivel global en un proceso de cambio climático, el IPCC (2014), ha determinado categóricamente que la temperatura de la atmósfera se viene incrementando con una notoria influencia antrópica produciendo cambios climáticos no observados en miles de años. Entre 1880 y 2012 la temperatura superficial terrestre y marina subió entre 0.65 a $1.06^{\circ} \mathrm{C}$, más recientemente, entre 2006 y 2015 la temperatura de la atmósfera sufrió un incremento de $0.87^{\circ} \mathrm{C}$ en promedio en comparación con el período preindustrial entre 1850 y 1900 . Para el período 2081-2100 se estima un calentamiento atmosférico global mayor a $1.5^{\circ} \mathrm{C}$ y en las zonas tropicales, de entre 1 y $2^{\circ} \mathrm{C}$.

Los glaciares peruanos son considerados de tipo tropical o temperados y son muy sensibles a las variaciones en la temperatura atmosférica. Desde el inicio de su monitoreo en 1962 hasta el año 2016 se ha perdido el 54\% de la superficie glaciar es decir $1,284.95 \mathrm{~km}^{2}$ (INAIGEM, 2018). Una consecuencia de esta reducción es la formación de lagunas glaciares habiéndose reportado entre 1980 y el 2003 la formación de 996 lagunas (Autoridad Nacional del Agua-ANA, 2014). Este escenario tiende a agravarse dadas las proyecciones de calentamiento atmosférico dando lugar al incremento de los peligros asociados a la desestabilización de los glaciares y la aparición e incremento del volumen de las lagunas de origen glaciar una de las cuales es Palcacocha cuyo volumen 34 veces entre 1974 y el 2016 (INAIGEM, 2018).

El 13 de diciembre de 1941 la ciudad de Huaraz fue parcialmente destruida por un aluvión originado en la laguna Palcacocha ubicada a $23 \mathrm{~km}$ al Este de la ciudad de Huaraz en la quebrada Cojup, subcuenca del río Quillcay. El fenómeno ocasionó también la pérdida de miles de vidas y la destrucción de infraestructura aguas abajo a lo largo del cauce del río Santa en el Callejón de Huaylas (Wegner, 2014).

Desde 1941 la población de la ciudad de Huaraz ha crecido ostensiblemente, en 1941 se estimaba en 34,998 habitantes, en 1981 llegó a 44,810, en 1993 a 66,890, el año 2007 se censaron 100,930 pobladores y el año 2015 se superaron los 120,000 habitantes (INEI, 2018). El crecimiento poblacional ha dado lugar consecuentemente al crecimiento urbano y urbano marginal, para el caso de la ciudad de Huaraz, de manera desordenada y no planificada permitiendo la ocupación de áreas expuestas a diversos peligros, destacando los de origen glaciar, según lo observado, se espera que la población y las zonas urbano marginales vulnerables sigan creciendo (INDECI, 2013).

Un aspecto importante para la gestión del territorio y la gestión de riesgos en el contexto mencionado es contar con información sobre la evolución histórica y futura del crecimiento de la ciudad para integrarla con información sobre los riesgos existentes. Actualmente, las herramientas geomáticas como los sistemas de percepción remota, sistemas de procesamiento de imágenes, sistemas de información geográfica (SIG) y software de programación $\mathrm{y}$ modelamiento especializado son fundamentales para generar información espacial sobre todo en lo relacionado a la gestión de riesgos (Huggel, 2004) y están disponibles tanto en plataformas gratuitas como comerciales.

Las herramientas geomáticas tienen la capacidad de procesar imágenes de sensores remotos como fotografías aéreas e imágenes satelitales multiespectrales a partir de las cuales es posible obtener información multitemporal sobre la cobertura de suelo, modelos de elevación digital, etc. (Chuvieco, 2010) la que posteriormente es empleada por los sistemas de información geográfica para efectuar operaciones de análisis espacial y modelamiento (Bosque Sendra \& García, 2013) entre las que se encuentran aquellas orientadas a analizar la tendencia espacial mediante el análisis de la distribución de elementos geográficos (Mitchell, 2005); y los procesos de cambio de uso de suelos existiendo dos grandes grupos de modelos: basados en reglas/ procesos y empírico-estadísticos. Los primeros, como los autómatas celulares buscan replicar el proceso analizado hasta cierto límite, poseen buenas capacidades para procesar la dinámica temporal, pero tienen limitaciones en el manejo de variables socioeconómicas. Los del segundo grupo emplean información histórica, así como características espaciales como posibles controladores del proceso analizado para aplicar técnicas de regresión múltiple espacial. Este tipo de modelos permite integrar mejor a los análisis variables socioeconómicas (Nong \& Du, 2011). La regresión logística es una técnica ampliamente utilizada en el análisis predictivo del crecimiento urbano pues permite modelar la probabilidad de que una variable binaria, como la cobertura urbana, tome un valor de 0 o 1 (falso o verdadero) en función a un conjunto de variables explicativas independientes (Nong \& Du, 2011).

La presente investigación analiza la tendencia espacial histórica de crecimiento de la ciudad de Huaraz en base a fotografías aéreas y satelitales de los años 1948, 1962, 1977, 2001 y 2018 y efectúa un análisis prospectivo de las nuevas áreas urbanas/pobladas potenciales generando información útil para la toma de decisiones en materia de gestión del territorio, planificación urbana y gestión de riesgos.

\section{MÉTODOS}

\section{1. Área de estudio}

Corresponde a la ciudad de Huaraz ubicada en las coordenadas 222,365E - 8'945,540S a una altitud de 3,065 m s.n.m., así como su periferia dentro de una zona de análisis con un área de $25.58 \mathrm{~km}^{2}$. Políticamente pertenece al departamento de Ancash, provincia de Huaraz y comprende los distritos de Huaraz e Independencia divididos por el río Quillcay afluente del río santa ubicado al Oeste de la ciudad (ver Figura 1).

\subsection{Sistema de referencia espacial y escala}

Se empleó el World Geodetic System 1984 (WGS84) - Zona 18 Sur en coordenadas Universal Transversal Mercator (UTM). El código European Petroleum Survey Group (EPSG) empleado fue 32718. La escala de trabajo fue 1:10,000 sobre un mapa base a nivel de manzanas obtenido del Plano Básico Catastral de la ciudad de Huaraz año 2004 y las cartas topográficas 19h-II-SE, 19i-III-SO, 20h-I-NE, 20i-IV-NO a escala 1:25,000. 


\subsection{Mapeo de la cobertura urbana}

Para analizar la evolución multitemporal de la cobertura urbana o poblada de la ciudad de Huaraz y su periferia de emplearon las siguientes fuentes de información:

- Fotografías aéreas 2524-53 y 2524-91 a escala 1:30,000 provista por el Área de Evaluación de Glaciares y Lagunas de la Autoridad Nacional del Agua, capturadas por el Instituto Geográfico Militar el 15/8/1948.

- Fotografía aérea 14241 a escala 1:40,000 provista por el Instituto Geográfico Nacional, capturada por USAF el 15-6-1962.

- Fotografía DZB1213-500232L005001 USGS EROS con resolución espacial de 20 a 30 pies capturada por el Sistema Satelital Keyhole (KH) el 04-10-1977, obtenida de la plataforma EarthExplorer.

- Fotografía aérea 2C-10A-53 a escala 1:25,000 capturada por la Mina Pierina - Barrick el 17-072001.

- Fotografías satelitales con una resolución espacial de un metro obtenidas de la plataforma Google Earth capturadas en octubre del año 2003 y agosto del año 2018.

Cada una de estas imágenes pancromáticas posee diferentes características geométricas y escalas y formatos por ello se efectuó una corrección geométrica empleando software geomático de procesamiento de imágenes sobre una zona de análisis rectangular de $25.6 \mathrm{~km}^{2}$ que comprende la ciudad de Huaraz y su periferia. La corrección aplicada fue de imagen a imagen de tipo spline (Donato \& Belongie, 2002) tomando como base la fotografía satelital del año 2018 cuyo ajuste espacial fue controlado sobre el mapa base mediante 72 puntos, obteniendo un Error Medio Cuadrático (RMSE) de $2.3 \mathrm{~m}$ valor inferior a los $3.1 \mathrm{~m}$ del RMSE máximo permisible para la escala de trabajo. Se emplearon en total 2,174 puntos de corrección con RMSE inferiores a un metro. Empleando sistemas de información geográfica (SIG) y técnicas de corrección e interpretación visual de imágenes (Chuvieco, 2010), se procedió a vectorizar los polígonos correspondientes a las zonas urbanas /pobladas para los años 1948, 1962, 1977, 2001 y 2018, aplicando las correspondientes correcciones topológicas.

\subsection{Tendencia espacial de crecimiento urbano}

Mediante el empleo de software SIG se aplicaron técnicas de medición de la distribución geográfica de los polígonos urbanos/poblados de cada año, para ello, se creó una nube aleatoria de puntos dentro de los polígonos teniendo como criterio una separación mínima de $2.24 \mathrm{~m}$ y un área mínima de $5 \mathrm{~m}^{2}$. Las capas de puntos densos se usaron para determinar los centros medios, que son puntos representativos de las coordenadas promedio de todos los puntos en cada capa anual, a través de las siguientes ecuaciones:

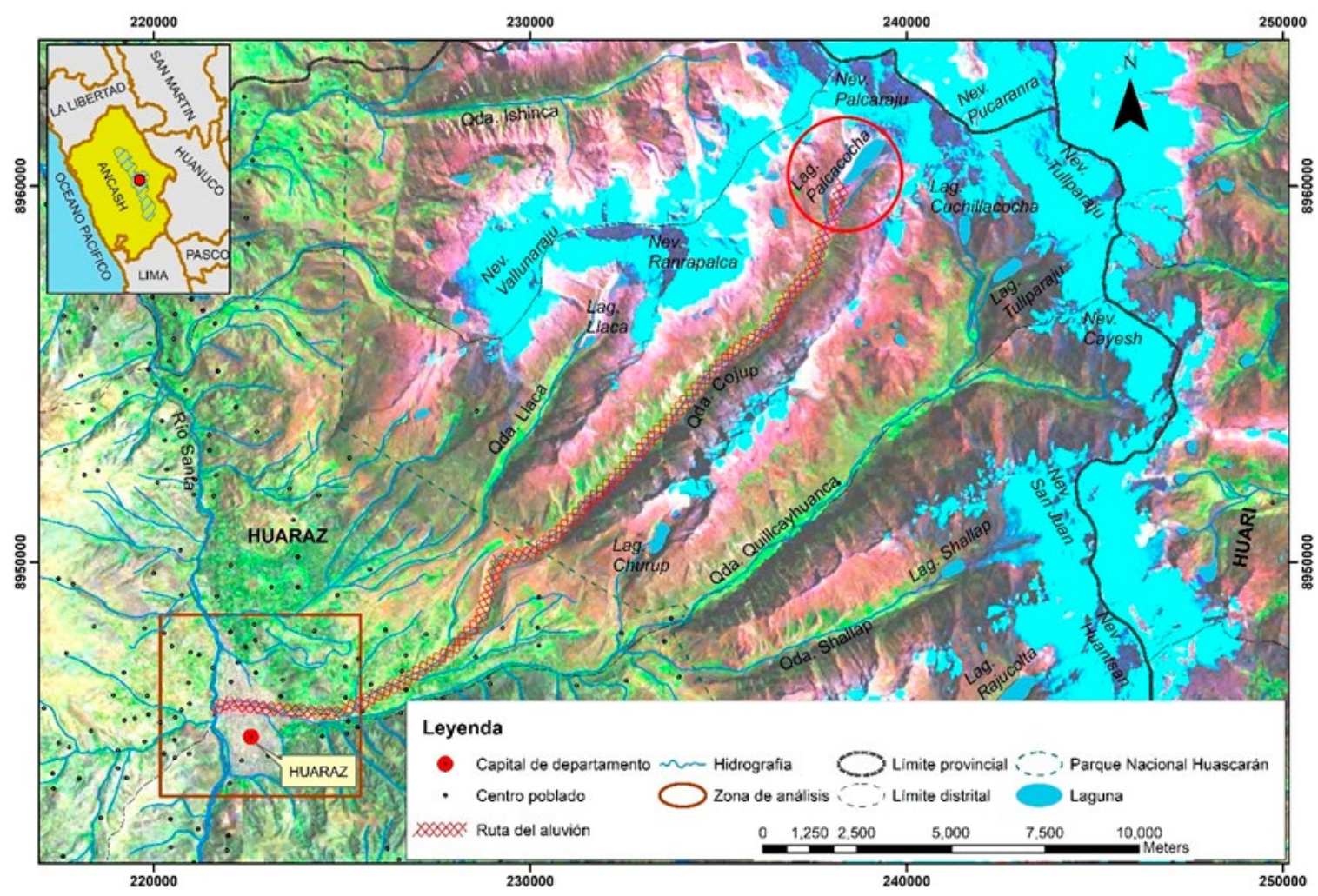

Figura 1. Mapa de ubicación de la ciudad de Huaraz y la zona de análisis. 


$$
\bar{X}=\frac{\sum_{i} X_{i}}{n} \quad \bar{Y}=\frac{\sum_{i} Y_{i}}{n}
$$

$X_{i}$ e $Y_{i}$ son las coordenadas de cada punto y $n$, su número. Las elipses de desviación estándar de cada año fueron también calculadas para evaluar la tendencia en la dirección de crecimiento de la ciudad. Las ecuaciones que controlan este procedimiento son:

$$
S D_{x}=\sqrt{\frac{\sum_{i}\left(X_{i}-\bar{X}\right)^{2}}{n}} \quad S D_{y}=\sqrt{\frac{\sum_{i}\left(Y_{i}-\bar{Y}\right)^{2}}{n}}
$$

$X_{i}$ e $Y_{i}$ son las coordenadas de los puntos, $n$ es el número de puntos, $\bar{X}$ e $\bar{Y}$ son las coordenadas promedio (Mitchell, 2005). Los centros medios y elipses su analizaron visualmente en conjunto para determinar la tendencia espacial histórica de crecimiento de la ciudad.

\subsection{Variables espaciales}

Es necesario contar con variables espaciales, conocidas también como drivig factors (Achmad et al., 2015) o fuerzas conductoras (Rojas \& Plata, 2010); que permitan modelar y explicar el crecimiento urbano/poblado de la ciudad. Las variables se procesaron como capas ráster de un metro de resolución espacial, fueron obtenidas empleando técnicas geomáticas de análisis espacial mediante SIG, de estas fueron:

- Distancia medida en metros a partir del centro medio multianual.

- Dirección, medida en grados sexagesimales a partir del centro medio multianual, siendo $0^{\circ}$ la dirección Norte.

- Pendiente, medida en porcentaje a partir del modelo de elevación digital (DEM).

- $\quad$ Altitud, medida en m.s.n.m. a partir del DEM ALOS PALSAR AP_27249_FBS_F6990_RT1.tif

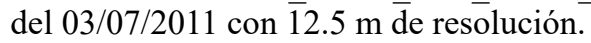

- Densidad de puntos, medida en puntos $/ \mathrm{km}^{2}$. Es una variable ficticia de apoyo que muestra el número de puntos en un radio de $100 \mathrm{~m}$.

\subsection{Regresión logística}

Tomando las variables espaciales como fuerzas conductoras del crecimiento urbano/poblado de la ciudad de Huaraz y como variables independientes, y la cobertura urbana/ poblada correspondiente al año 2018 como variable dependiente, se ejecutó la regresión logística espacial técnica extensamente utilizada en casos en que la variable dependiente es binaria, es decir toma el valor de 1 cuando para zonas urbanas y 0 para zonas en caso contrario (Rojas \& Plata, 2010). Para este fin inicialmente fue necesario verificar la no colinealidad de las variables independientes a través del análisis de correlación de Pearson, posteriormente se ingresan las variables independientes y dependiente a un software geomático para ejecutar la regresión logística cuyo resultado es la probabilidad de que la variable dependiente sea 1, según la siguiente ecuación:

$$
P_{(y=1 \mid X)}=\frac{e^{\sum B X}}{1+e^{\sum B X}}
$$

$P$ de que la probabilidad, $B$ representa los parámetros $(b 0, b 1, b 2, b 3, \ldots . b k)$ y $X$ representa las variables independientes $(x 0, x 1, x 2, x 3, \ldots . x k)$. Para su solución, esta ecuación debe ser linearizada a través de una transformación logística (Eastman, 2012) conocida como logit:

$$
\operatorname{Ln}\left(\frac{P}{1-P}\right)=b_{0}+b_{1} x_{1}+b_{2} x_{2}+\cdots+b_{k} x_{k}+\text { error }
$$

Los coeficientes obtenidos fueron estandarizados para su análisis comparativo (Rojas \& Plata, 2010):

$$
\text { B estandarizado }=\left(B^{*} \text { Desviación estándar }\right) /\left(\pi^{2} / 3\right)
$$

De la ejecución de la regresión logística se obtienen un conjunto de estadísticos que permiten evaluar el nivel de ajuste de los resultados como en pseudo $\mathrm{R}^{2}$ cuyo valor debe tender a 1 y odds ratio cuyo valor debe ser positivo y alejado de 1 . Se efectuó también el análisis denominado Relative Operating Characteristic (ROC) (Eastman, 2012) a fin de validar el modelo de probabilidad obtenido en función al mapa binario del año 2018, se espera que su valor sea cercano a 1 y mayor que 0.5 .

\section{RESULTADOS Y DISCUSIÓN}

\subsection{Crecimiento urbano de la ciudad de Huaraz}

La ciudad de Huaraz creció vertiginosamente luego de acontecido el aluvión de 1941, como se puede apreciar en la Tabla 1 y Figura 2.

Tabla 1. Evolución histórica de las áreas urbanas/pobladas de la ciudad de Huaraz

\begin{tabular}{cccc}
\hline Año & Área (ha) & \multicolumn{2}{c}{ Incremento } \\
\hline 1948 & 111.0 & ha & $\%$ \\
1962 & 157.9 & 46.9 & $42.2 \%$ \\
1977 & 339.5 & 181.6 & $115.0 \%$ \\
2001 & 588.5 & 249.0 & $73.3 \%$ \\
2018 & 774.7 & 186.2 & $31.6 \%$ \\
\hline
\end{tabular}

En 1948, seis años después del aluvión, el 86\% de la zona urbana/poblada se localizaba al Sur del río Quillcay, conformando el núcleo histórico de Huaraz, no existía aun el actual distrito de Independencia.

En 1962, el 76\% de las áreas urbanas/pobladas de la ciudad se localizaban en el actual distrito de Huaraz, al Sur del río Quillcay; empezó a crecer el barrio de Centenario, al Norte de la ciudad en el actual distrito de Independencia. Después del terremoto de 1970, en la escena de 1977 se evidencia el mayor crecimiento urbano en términos porcentuales $(115 \%)$ sobre todo al Norte del río Quillcay en barrios como Centenario, Patay y Nicrupampa, ocupando el $38 \%$ de la ciudad. En el sector Sur de Huaraz, se desarrollaron sobre todo los barrios de Pedregal y Villón. Para el año 2001 se registró el mayor incremento de nuevas 

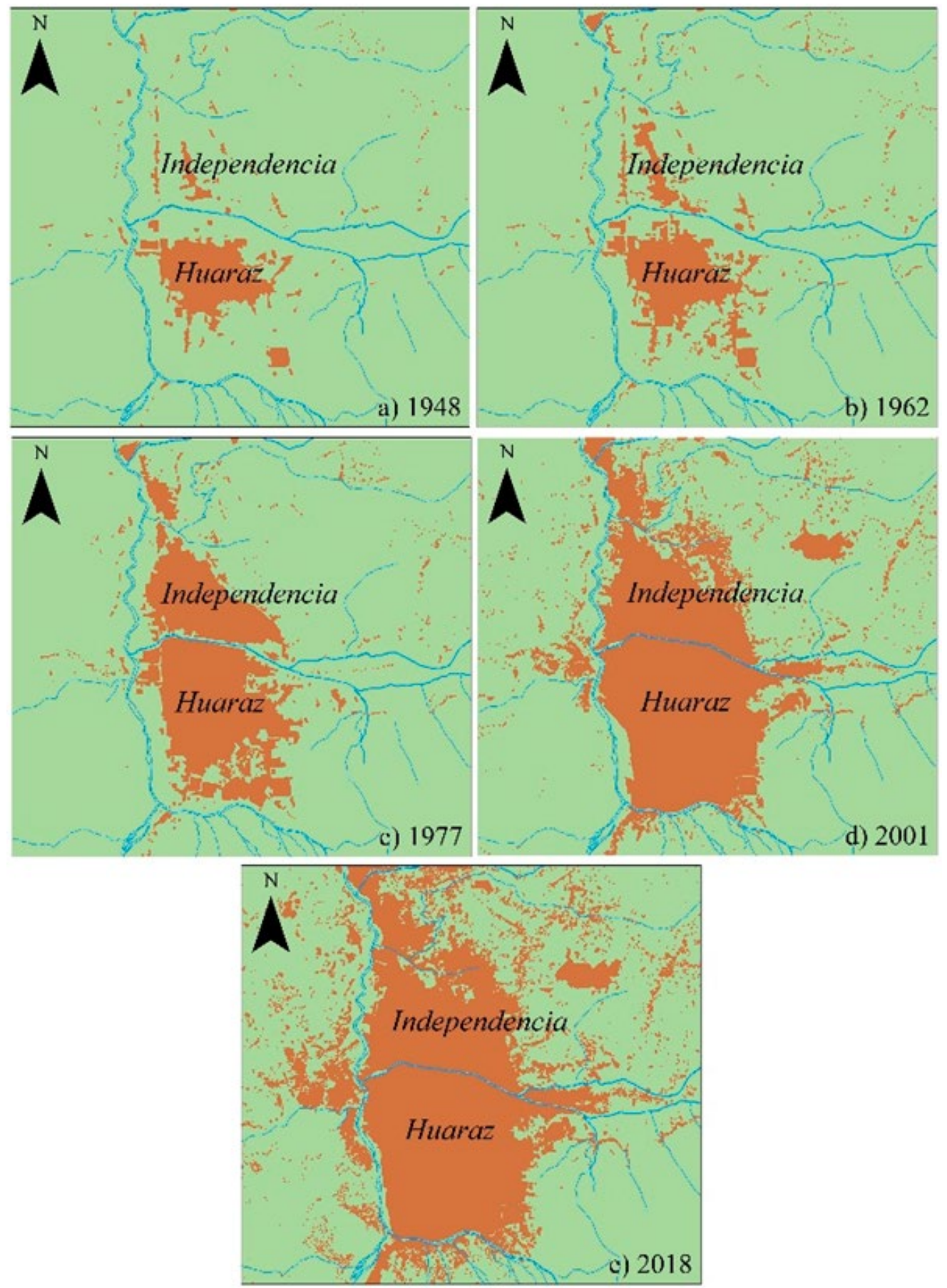

Figura 2. Evolución espacial multianual de las áreas urbanas/pobladas

áreas urbanas/pobladas (249 ha), continuó en este período el crecimiento de la ciudad al Norte del río Quillcay, el $45 \%$ se ubicó en el ya existente distrito de Independencia consolidándose el crecimiento de barrios como Shancayán, Los Olivos, Patay, Quinuacocha y Nueva Florida. Al Sur de la ciudad crecían los barrios de Tacllán, Villón, Challhua, Río Seco y Pedregal. Para el 2001, la zona afectada por el aluvión de 1941 estaba ya densamente poblada.

Hasta el año 2018, ya el 52\% de la ciudad se localizaba en el distrito de Independencia y continuó el crecimiento al Sur de barrios como Cochac, Los Olivos, Shancayán y
Nueva florida y al Norte, Tacllán, Río Seco y Challhua; así como de núcleos poblados dispersos en la zona rural (ver Figura 3 referencia a los barrios de Huaraz).

En general, el crecimiento de los nuevos barrios en la periferia de la ciudad fue desordenado y carente de planificación, con construcciones precarias, falta de acceso a servicios básicos y alta vulnerabilidad frente a peligros físicos.

En el período de 14 años entre 1948 y 1962 se registró un crecimiento promedio de 3.3 ha/año, tasa que 


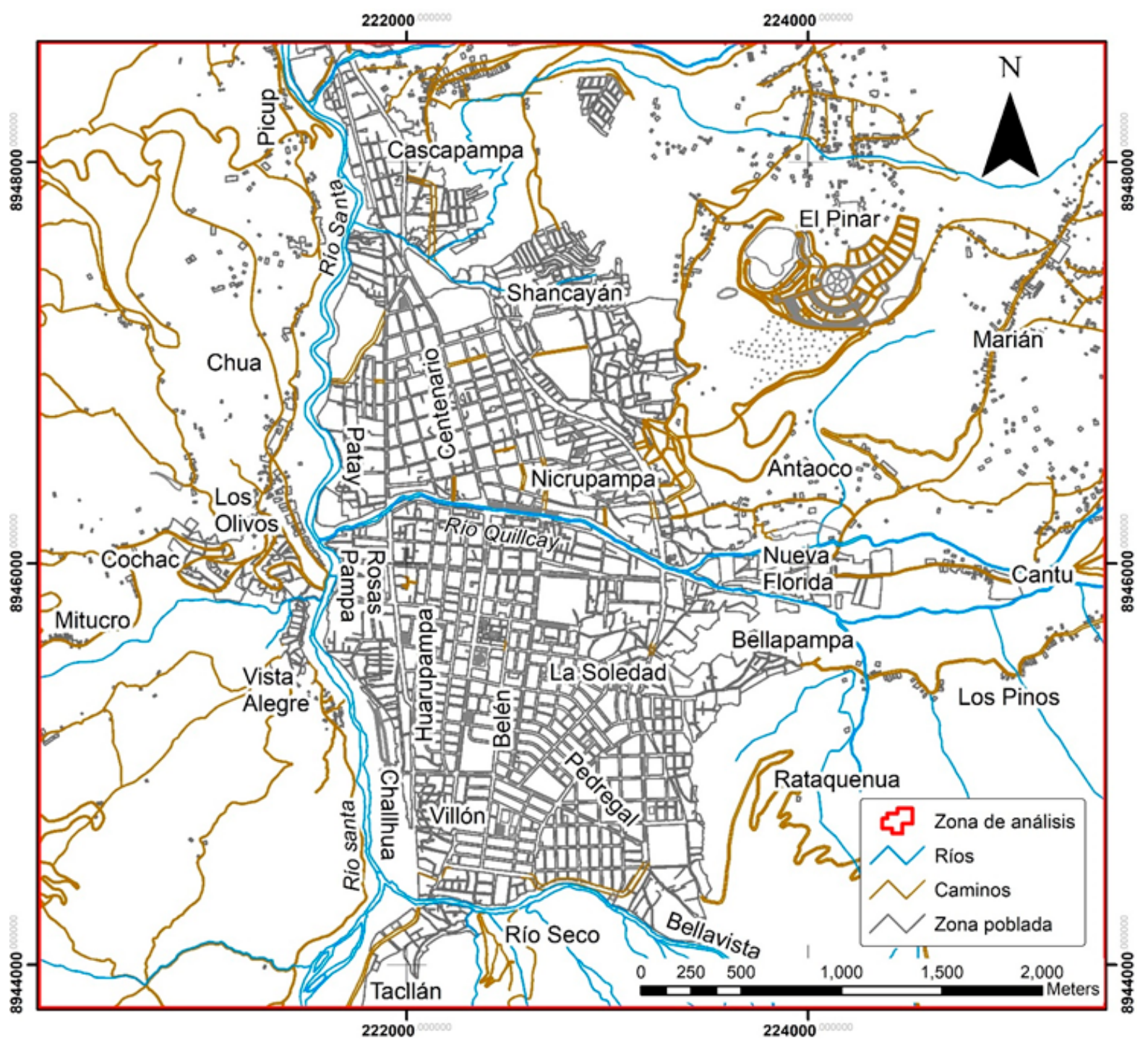

Figura 3. Distribución de los principales barrios de la ciudad de Huaraz dentro de la zona de análisis

se incrementó sensiblemente en el período 1962-1977 de 15 años llegando a 12.1 ha/año. En el período 1977-2001 de 24 años, la tasa promedio de crecimiento bajó a 10.4 ha/ año, incrementándose a 11 ha/año en el período de 17 años entre el 2001 y el 2018.

\subsection{Tendencia espacial del crecimiento urbano}

Se evidencia que los centros medios correspondientes a los cinco años en análisis han evolucionado en dirección Norte desde 1948 año en el que el centro medio se encontraba en el núcleo histórico de la ciudad (ver Tabla 2 y Figura 4). El año 2001 la tendencia de los centros medios de años anteriores se desplazamiento hacia el Este evolucionando hacia el Norte para el 2018.

Complementariamente, el análisis de las elipses de desviación estándar (SD) refuerza lo antes observado, el tamaño y excentricidad de las elipses demuestra el continuo crecimiento de la ciudad sobre todo en el período 19772001, en dirección predominantemente Norte (ver Tabla 3 y Figura 4).

Tabla 2. Evolución de los centros medios anuales

\begin{tabular}{rrrrr}
\hline Año & UTM "X" & UTM "Y" & $\begin{array}{c}\text { Desplaz. } \\
(\mathrm{m})\end{array}$ & Direcc. \\
\hline 1948 & 222538.5 & 8945683.8 & & \\
1962 & 222543.9 & 8945826.9 & 143.2 & Norte \\
1977 & 222522.2 & 8945981.1 & 155.7 & Norte \\
2001 & 222593.8 & 8946051.4 & 100.4 & Noreste \\
2018 & 222591.8 & 8946155.2 & 103.8 & Norte \\
Interan. & 222558.0 & 8945939.7 & & \\
\hline
\end{tabular}




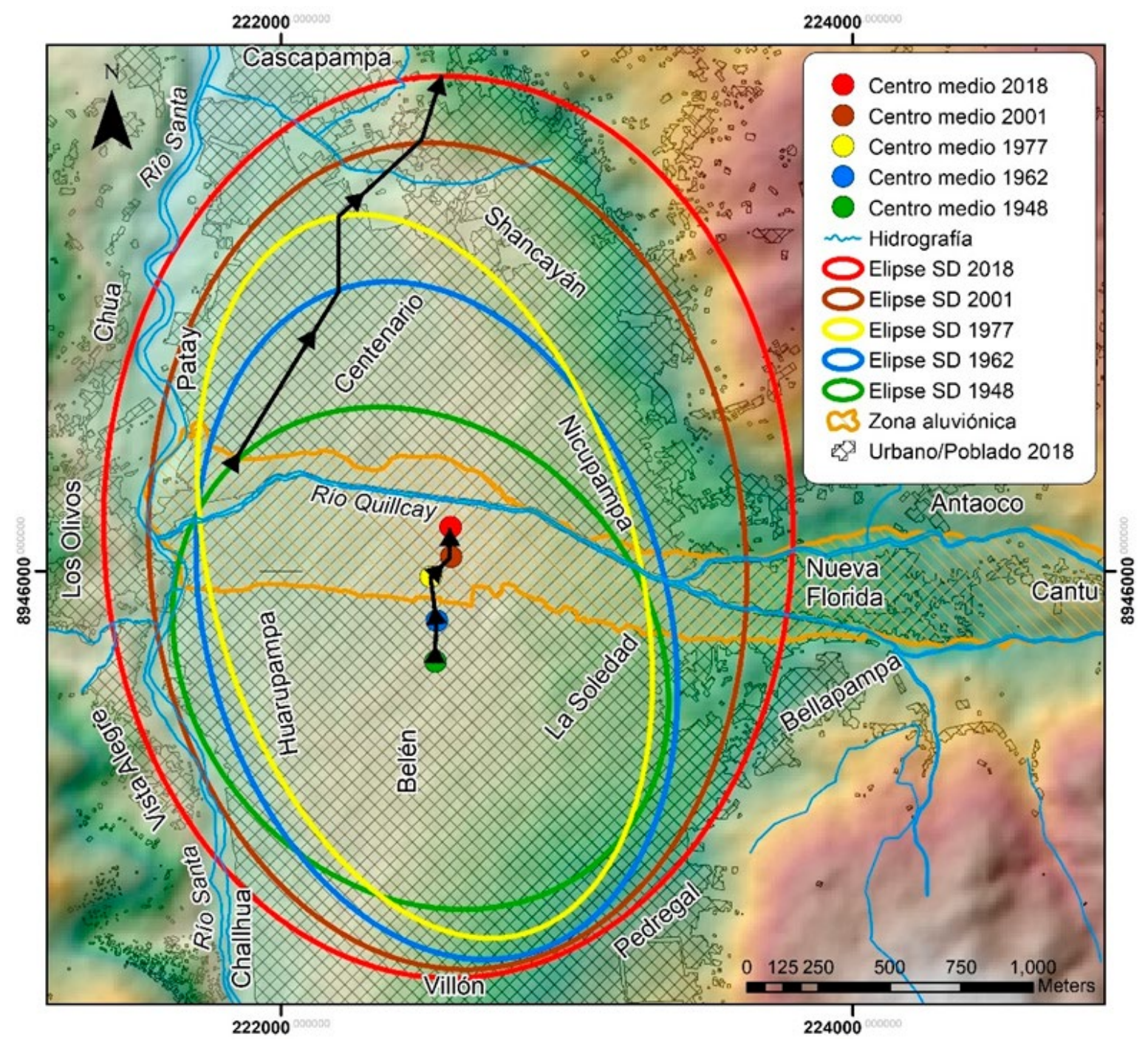

Figura 4. Tendencia espacial del crecimiento de la ciudad de Huaraz

Tabla 3. Evolución de las elipses SD por año

\begin{tabular}{|c|c|c|c|}
\hline Año & Centro $\mathrm{X}, \mathrm{Y}$ & $\begin{array}{c}\text { Distancia estándar } \\
X, Y \\
\end{array}$ & Rotación $\left({ }^{\circ}\right)$ \\
\hline 1948 & $\begin{array}{c}222487.3 \\
8945695.1\end{array}$ & $\begin{array}{l}796.0 \\
944.4\end{array}$ & 137.2 \\
\hline 1962 & $\begin{array}{c}222543.9 \\
8945826.9 \\
\end{array}$ & $\begin{array}{l}809.7 \\
1208.9 \\
\end{array}$ & 165.0 \\
\hline 1977 & $\begin{array}{c}222502.5 \\
8945982.2\end{array}$ & $\begin{array}{c}744.2 \\
1297.8\end{array}$ & 164.6 \\
\hline 2001 & $\begin{array}{c}222581.6 \\
8946052.8\end{array}$ & $\begin{array}{l}1042.9 \\
1449.1\end{array}$ & 174.9 \\
\hline 2018 & $\begin{array}{c}222584.0 \\
8946154.4\end{array}$ & $\begin{array}{l}1206.0 \\
1578.1\end{array}$ & 179.3 \\
\hline
\end{tabular}

La evolución de la rotación de los ejes mayores de las elipses SD, así como de la dirección de crecimiento respecto al centro medio interanual en cada período, muestra inequívocamente la tendencia de crecimiento en dirección Noreste, es decir hacia los sectores de Shancayán, Huanchac y Antaoco, sin embargo, es apreciable también el crecimiento en los sectores Este y Oeste de la ciudad en los barrios de Nueva Florida y Los Olivos. Entre 1948 y 2018 las áreas urbanas/pobladas de Huaraz crecieron también ocupando paulatinamente territorios con mayores altitudes y pendientes.

\subsection{Resultado del modelo de regresión logística}

Del análisis se correlación de Pearson aplicado entre las variables independientes que se emplearon en la regresión logística se obtuvieron coeficientes inferiores al umbral de 0.8 (Rojas \& Plata, 2010) (ver Tabla 4). Se excluyó la variable Luego de corridas iniciales del modelo de regresión logística, se excluyó del análisis la variable densidad de puntos por su alta correlación con la cobertura urbana del año 2018 y la variable dirección al centro medio interanual pues distorsionaba espacialmente los resultados del modelo.

Tabla 4. Correlación de las variables independientes

\begin{tabular}{cccc}
\hline Variable & Pendiente & Altitud & Distancia \\
\hline Pendiente & 1.000 & --- & --- \\
Altitud & 0.366 & 1.000 & --- \\
Distancia & 0.271 & 0.636 & 1.000 \\
\hline
\end{tabular}


La Tabla 5 muestra los resultados del modelo de regresión logística espacial aplicado. Se logró un buen nivel de ajuste sustentado en el pseudo $\mathrm{R}^{2}$ de 0.4478 , un odds ratio de 32.6 que indica una elevada probabilidad de que los valores modelados coincidan con los observados y un valor ROC de 0.916. Los coeficientes resultantes del modelo fueron estandarizados para su comparación, se puede observar que todas las variables tienen signo negativo, es decir su relación es inversa con la variable dependiente, a mayor altitud, mayor pendiente y distancia al centro medio interanual; menor probabilidad de localización de nuevas áreas urbanas/pobladas.

Tabla 5. Resultados de la regresión logística

\begin{tabular}{ccccc}
\hline Variables & Promedio & $\begin{array}{c}\text { Desv. } \\
\text { estándar }\end{array}$ & Coef. & $\begin{array}{c}\text { Coef. } \\
\text { estándar. }\end{array}$ \\
\hline Interseptor & ------ & ------ & 27.979 & ------ \\
Altitud & 3201.094 & 111.227 & -0.008 & -0.260 \\
Distancia & 1962.548 & 753.522 & -0.002 & -0.412 \\
Pendiente & 22.325 & 16.011 & -0.065 & -0.318 \\
\hline
\end{tabular}

La variable con mayor influencia en el establecimiento de nuevas áreas urbanas/pobladas es la distancia al centro medio interanual, es decir la población prefiere establecerse lo más cerca posible de la zona céntrica de la ciudad de Huaraz. En segundo y tercer nivel de importancia de encuentran la pendiente y la altitud.

Espacialmente (ver Figura 5), sin considerar las áreas urbanas del año 2018, los resultados muestran que más del $50 \%$ de la zona de análisis posee bajas probabilidades de albergar nuevas áreas urbanas/pobladas (entre 0 y 0.2 ), sin embargo, existen 382 ha (15\% de la zona de análisis) con probabilidades superiores a 0.2 que consideramos de especial interés (ver Tabla 6).

Estas áreas están localizadas en la periferia de Huaraz, en los sectores o barrios de El Pinar, Cascapampa, Shancayán, Bellapampa, Chua, Picup, Vista Alegre, Mitucro, Cochac, Los Olivos, Tacllán, Pedregal, Challhua y Río Seco; destacando los barrios de Nueva Florida y Antaoco, con áreas comprendidas dentro de la zona aluviónica (ver Figura 5).

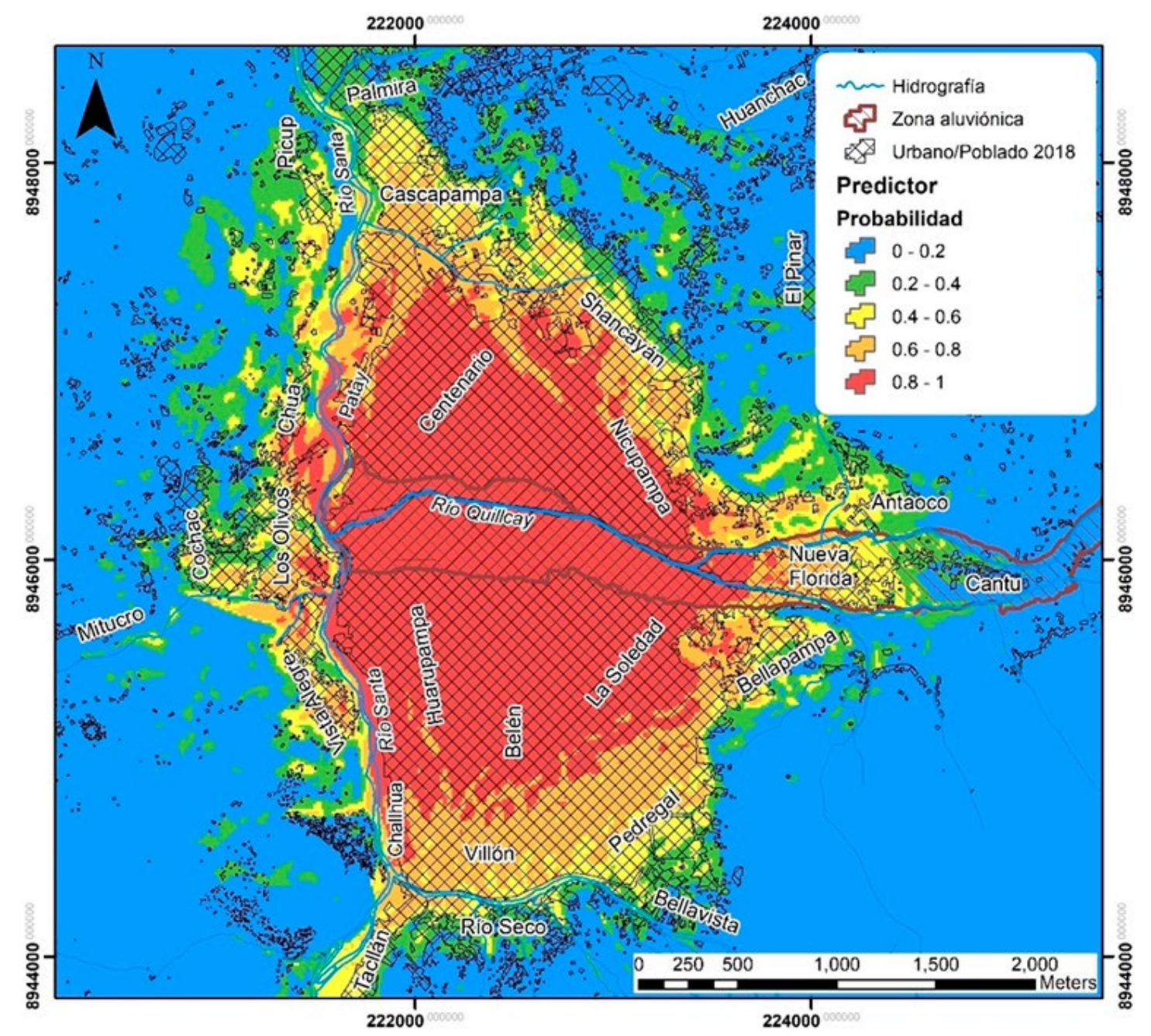

Figura 5. Mapa de probabilidades de crecimiento urbano de la ciudad de Huaraz 
Tabla 6. Probabilidades de crecimiento urbano de Huaraz por áreas (ha) en la zona de análisis

\begin{tabular}{ccc}
\hline Probabilidad & Área (ha) & $\%$ \\
\hline Urbano 2018 & 774.7 & $30.3 \%$ \\
$0.0-0.2$ & 1401.8 & $54.8 \%$ \\
$0.2-0.4$ & 201.3 & $7.9 \%$ \\
$0.4-0.6$ & 81.5 & $3.2 \%$ \\
$0.6-0.8$ & 62.8 & $2.5 \%$ \\
$0.8-1.0$ & 36.3 & $1.4 \%$ \\
\hline Total & 2558.4 & $100.0 \%$ \\
\hline
\end{tabular}

\section{CONCLUSIONES}

Las herramientas geomáticas para el procesamiento digital de imágenes de sensores remotos y los sistemas de información geográfica (SIG), han demostrado ser herramientas fundamentales para los estudios territoriales como el abordado en la presente investigación; permiten en conjunto, producir resultados óptimos en términos de tiempo y precisión útiles para la toma de decisiones.

Mediante las herramientas de análisis espacial y modelamiento de los SIG fue posible analizar el crecimiento histórico de las zonas urbanas/pobladas de la ciudad de Huaraz y su tendencia espacial de crecimiento logrando determinar que para el año 2018 la ciudad creció 598\% con respecto a 1948 en dirección Noreste sobre todo en el distrito de Independencia, con una tasa promedio de $9.2 \mathrm{ha} /$ año para este período, ocupando paulatinamente territorios ubicados a mayores altitudes y más accidentados, inclusive en áreas expuestas a peligros físicos como el aluvión acontecido en 1941. Según lo observado, esta tendencia de crecimiento continuará en los próximos años dada la débil gestión territorial imperante.

Los resultados del modelo de regresión logística muestran que los barrios en la periferia de la ciudad de Huaraz continuarán su crecimiento inclusive en los núcleos poblados dispersos de la zona rural observados entre los años 2001 y 2018, así como en áreas comprometidas desde un punto de vista de gestión de riesgos tal es el caso de los barrios de Nueva Florida y Antaoco con áreas localizadas en la zona aluviónica, Challhua ubicado en el antiguo cauce del río Santa, Río Seco y Los Olivos ubicados en áreas muy accidentadas; en todos estos casos se observa también precariedad en la ocupación de territorio, falta se servicios y sistemas constructivos vulnerables.

La presente investigación aporta una experiencia metodológica e información sobre la dinámica de crecimiento de la ciudad de Huaraz que se espera sea de utilidad para dar sustento a la toma de decisiones orientadas a mejorar la planificación de crecimiento urbano y la gestión de riesgos.

\section{AGRADECIMIENTOS}

Las siguientes instituciones y personas contribuyeron al desarrollo de la presente investigación:
- Consejo Nacional de Ciencia, Tecnología e Innovación Tecnológica - CONCYTEC.

- Área de Evaluación de Glaciares y Lagunas AEGL de la Autoridad Nacional del Agua. Ing. Alejo Cochachin Rapré.

- Instituto Geográfico Nacional - IGN. Cmdt. EP Víctor Huamán Maguiña.

- CARE Perú. Ing. Karen Price Ríos.

- Asociación Waras. Bibl. Gíber García Álamo.

- Arq. Steven A. Wegner.

\section{REFERENCIAS}

Achmad, A., Hasyim, S., Dahlan, B., \& Aulia, D. N. (2015). Modeling of urban growth in tsunami-prone city using logistic regression: Analysis of Banda Aceh, Indonesia. Applied Geography, 62, 237-246. https://doi.org/10.1016/j. apgeog.2015.05.001

Autoridad Nacional del Agua-ANA. (2014). Inventario Nacional de Glaciares y Lagunas. Autoridad Nacional Del Agua. Unidad de Glaciología y Recursos Hidricos, 19. https:// repositorio.ana.gob.pe/bitstream/handle/20.500.12543/199/ ANA0000015.pdf? sequence $=4 \&$ isAllowed $=y$

Bosque Sendra, J., \& García, R. C. (2000). El Uso de los Sistemas de Información Geográfica en la Planificación Territorial. Anales de Geografia de La Universidad Complutense, 20, 49-67. https://revistas.ucm.es/index.php/AGUC/article/ view/AGUC0000110049A/31281

Chuvieco, E. (2010). Teledeteccion Ambiental: La Observacion De La Tierra Desde Espacio. https://www.researchgate. net/publication/259011213_Teledeteccion_Ambiental_La_ Observacion_de_la_Tierra_Desde_el_Espacio

Donato, G., \& Belongie, S. (2002). Approximate thin plate spline mappings. Lecture Notes in Computer Science (Including Subseries Lecture Notes in Artificial Intelligence and Lecture Notes in Bioinformatics), 2352, 21-31. https://doi. org/10.1007/3-540-47977-5_2

Eastman, J. R. (2012). IDRISI Selva. Guía de IDRISI para SIG y el Procesamiento de Imágenes. IDRISI Selva Manual, January, 1-324. https://clarklabs.org/wp-content/uploads/2016/10/ IDRISI-Selva-Spanish-Manual.pdf

Huggel, C. (2004). Assessment of Glacial Hazards based on Remote Sensing and GIS Modeling [Geographisches Institut der Universität Zürich]. In Schriftenreihe Physische Geographie Glaziologie und Geomorphodynamik (Vol. 44). http://citeseerx.ist.psu.edu/viewdoc/ download?doi=10.1.1.132.1175\&rep=rep1\&type $=$ pdf

INAIGEM. (2017). Situación de los glaciares y ecosistemas de montana en el Perú (Dirección de Información y Gestión del Conocimiento (ed.); p. 108). Instituto Nacional de Investigación en Glaciares y Ecosistemas de Montaña. https://www.inaigem.gob.pe/wp-content/uploads/2019/04/ Informe-2018-v33-formato-digital.pdf

INDECI. (2013). Plan de prevención ante desastres: Usos del suelo y medidas de mitigación Ciudad de Huaraz. Instituto 
Nacional De Defensa Civil. https://sigrid.cenepred.gob.pe/ sigridv3/documento/232

Instituto Nacional de Estadística e Informática. (2017). Censos Nacionales 2017: XII de Poblacion, VII de Vivienda y III de Comunidades. https://www.inei.gob.pe/media/ MenuRecursivo/publicaciones_digitales/Est/Lib1530/libro. pdf

IPCC. (2014). Cambio Climático - Informe de Sintesis. Contribución de Los Grupos de Trabajo I, II y III Al Quinto Informe de Evaluación Del Grupo Intergubernamental de Expertos Sobre El Cambio Climático. https://www.ipcc.ch/ site/assets/uploads/2018/02/SYR_AR5_FINAL_full_es.pdf

Mitchell, A. (2005). ESRI Guide to GIS Analysis, Volume 2: Spatial Measurements and Statistics. https://esripress.esri. com/display/index.cfm?fuseaction=display\&websiteID $=86$ \&moduleID $=0$
Nong, Y., \& Du, Q. (2011). Geo-spatial Information Science Urban growth pattern modeling using logistic regression. Online) Journal, 1993-5153. https://www.tandfonline.com/ doi/abs/10.1007/s11806-011-0427-x

Rojas, C., \& Plata, W. (2010). Área Metropolitana de Concepción: Factores espaciales explicativos de su crecimiento urbano reciente (2001-2009) por medio de un Modelo de Regresión Logística Espacial. Actas I Congreso Internacional de Ordenamiento Territorial y Tecnologías de La Información Geográfica., 4, 1-12. http://faces.unah.edu.hn/decanato/ images/stories/PDF/Revista_Congreso_V2/area_ metropolitana_concepcion.pdf

Wegner, S. (2014). Lo que el Agua se Llevó. Dirección Desconcentrada de Cultura Áncash - MINAM - BID, 88. https://issuu.com/cultura-ancash/docs/lo_que_el_agua_se llevo_-_steven_we 